\title{
Efficacy of 4'-[methyl-11C] thiothymidine PET/CT before and after neoadjuvant therapy for predicting therapeutic responses in patients with esophageal cancer: a pilot study
}

\author{
Masatoshi Hotta ${ }^{1,4^{*}}$ (D) Ryogo Minamimoto ${ }^{1}$, Kazuhiko Yamada ${ }^{2}$, Kyoko Nohara², Daisuke Soma²,
}

Kazuhiko Nakajima', Jun Toyohara ${ }^{3}$ and Kei Takase ${ }^{4}$

\begin{abstract}
Background: 4'-[Methyl-11C] thiothymidine (4DST) has been introduced as a new cell proliferation imaging PET tracer that incorporates into DNA directly. The aim of this prospective study was to evaluate the efficacy of 4DST PET/CT for predicting responses to neoadjuvant therapy in patients with esophageal cancer comparing with FDG PET/CT.

Methods: Twenty-six patients who had pre- and post-therapeutic 4DST and FDG PET/CT and underwent esophagectomy following neoadjuvant therapy were used for the analysis. Based on pathological findings, patients were divided into two groups: non-responders and responders. The maximum standardized uptake value (SUVmax), metabolic tumor volume, total lesion glycolysis, and total lesion proliferation of the primary lesion were measured for FDG and 4DST PET.

Results: The pathological diagnosis revealed 16 responders and 10 non-responders. Non-responders showed significantly higher 4DST post-therapeutic SUVmax (post $S U V$ max) than responders, whereas FDG post $S U V$ max showed no statistically significant difference (non-responders vs. responders: 4DST, 6.7 vs. 3.3, $p=0.001$; FDG, 6.1 vs. 4.5, $p=0.11$ ). Responders showed a greater reduction in percentage changes of 4DST and FDG SUVmax ( $\triangle$ SUVmax) from baseline to post-therapeutic PET (non-responders vs. responders: 4DST, $-2.9 \%$ vs. $-56.7 \%, p<0.001$; FDG, $-36.3 \%$ vs. $-72.6 \%$, $p<0.001$ ). In ROC analysis, $\triangle S U V \max$ and ${ }_{\text {post }} S U V$ max with $4 D S T$ provided great diagnostic performance for predicting responses (area under the curve: 4DST $\Delta$ SUVmax $=0.92,4 D S T$ post $S U V \max =0.88$ ).
\end{abstract}

Conclusions: 4DST PET/CT has a great potential for predicting pathologic response to neoadjuvant therapy in patients with esophageal cancer; it may be slightly superior to that with FDG PET/CT.

Keywords: 4DST, Esophageal cancer, PET/CT, FDG, Therapeutic response

\footnotetext{
* Correspondence: masatoshihotta@yahoo.co.jp

${ }^{1}$ Division of Nuclear Medicine, Department of Radiology, National Center for

Global Health and Medicine, 1-21-1, Toyama, Shinjuku-ku, Tokyo 162-8655,

Japan

${ }^{4}$ Department of Diagnostic Radiology, Tohoku University Hospital, 1-1,

Seiryo-machi, Aoba-ku, Sendai, Miyagi 980-8574, Japan

Full list of author information is available at the end of the article
} 


\section{Background}

The prognosis of patients with advanced esophageal cancer continues to be poor, despite advances in management. Neoadjuvant chemotherapy or chemoradiation therapy before esophagectomy is a standard-of-care and commonly applied in clinical practice for locally advanced and operable esophageal cancer [1-3]. Once neoadjuvant therapy is completed, assessment of response is necessary [3]. When a persistent local lesion is indicated, esophagectomy is strongly recommended because the presence of residual tumor after neoadjuvant therapy in the resected specimen leads to shorter overall survival [3-5]. On the contrary, if there is no evidence of residual viable lesion, surveillance can be a possible option [3]. Generally, FDG PET/CT and/or contrastenhanced chest CT is used for the evaluation of treatment response of neoadjuvant therapy in esophageal cancer [3]. It is sometimes difficult to distinguish a viable residual tumor form reactive changes with a chest CT. In contrast, FDG PET/CT provides a more accurate diagnosis compared to that with chest $\mathrm{CT}$ due to its evaluation of metabolic activity. However, the value of FDG-PET/CT for evaluating response to neoadjuvant therapy in esophageal cancer is still controversial [6-8], so it is basically not recommended for the selection of patients for esophagectomy following neoadjuvant therapy [3].

Recently, Toyohara et al. developed 4' -[methyl-11C] thiothymidine (4DST) as a new DNA synthesis imaging agent $[9,10]$. Although 3 '-fluoro-3'-deoxythymidine
(FLT) has been established as a cell proliferation PET tracer, 4DST has advantages for proliferation measurement [11]. 4DST incorporates into DNA directly, whereas FLT does not incorporate into DNA and reflects salvage pathway of DNA synthesis [12]. We have previously reported on the great potential of 4DST PET/CT for proliferation imaging in malignancies such as lung cancer and renal cell carcinoma [13-15]. In addition, Hoshikawa et al. have reported that 4DST PET shows a higher prognostic value in patients with head and neck carcinoma compared to FDG PET [16].

These results suggest that 4DST PET can potentially predict a response to neoadjuvant therapy in esophageal cancer. The aim of this study was to evaluate the diagnostic value of 4DST for predicting response to neoadjuvant therapy in patients with esophageal cancer as compared to that with FDG.

\section{Methods \\ Patients}

This prospective study was approved by the institutional review board of our hospital, and written informed consent was obtained from all patients. We enrolled patients with biopsy-proven esophageal cancer. A total of 49 consecutive treatment-naïve patients underwent baseline 4DST and FDG PET/CT from August 2015 to September 2018 and were assessed for eligibility for this prospective study (Fig. 1). Among them, 11 patients were treated with definitive chemoradiation therapy (8 patients declined to undertake esophagectomy, and 3

$$
\begin{gathered}
\text { Treatment naïve patients with esophageal cancer who } \\
\text { underwent baseline 4DST and FDG PET/CT } \\
(n=49)
\end{gathered}
$$

Fig. 1 Flow diagram of patient selection 
patients were regarded as inoperable due to newly diagnosed lung and/or bone metastasis), 5 patients were treated with esophagectomy without neoadjuvant therapy, and 5 patients did not have 4DST and/or FDG PET/ CT after neoadjuvant therapy. In addition, 2 patients with adenocarcinoma were excluded because of the different biological entity between adenocarcinoma and squamous cell carcinoma. Therefore, we analyzed 26 patients with esophageal squamous cell carcinoma (23 men and 3 women, mean \pm SD age $66.4 \pm 9.7$ years). They all underwent esophagectomy following neoadjuvant therapy. Exclusion criteria for these 26 patients were (1) uncontrolled diabetes or (2) non-avid tumors on FDG or 4DST PET/CT, but no patients met these exclusion criteria. Patients' demographics including tumor markers are shown in Table 1. Regarding the regimen of neoadjuvant therapy, the chemotherapy consisted of fluoropyrimidine with platinum for all patients. The total radiation dose for the radiation therapy was 40.0 Gy $(n=11 / 12$ [91.7\%]) or $60 \mathrm{~Gy}(n=1 / 12$ [8.3\%]) delivered in daily fractions.

\section{DST-PET/CT examination}

The 4DST was synthesized using a previously described method [17]. All subjects fasted for $5 \mathrm{~h}$ before

Table 1 Patients demographics

\begin{tabular}{ll}
\hline Demographics & Number \\
\hline Patients & 26 \\
Sex, male & 23 \\
Mean age years (standard deviation) & $66.4(9.7)$ \\
Location & \\
Upper & 7 \\
Middle & 14 \\
Lower & 5 \\
Clinical T-stage & \\
CT2 & 2 \\
CT3 & 13 \\
CT4 & 11 \\
Clinical N-stage & \\
CNO & 6 \\
CN1 & \\
CN2 & 6 \\
CN3 & 13 \\
Neoadjuvant therapy & 12 \\
Chemotherapy & \\
Chemoradiotherapy & $12.2(0.2,8.6)$ \\
Tumor marker & \\
SCC (ng/mL) [median, (range)] & 14 \\
CEA (ng/mL) [median, (range)] & \\
\hline
\end{tabular}

receiving the intravenous injection of 4DST with a mean of $361 \pm 46 \mathrm{MBq}$ (pre-treatment) and $361 \pm 22$ $\mathrm{MBq}$ (post-treatment). PET/CT images were obtained from skull base to pelvis $40 \mathrm{~min}$ after intravenous injection and performed using either of cross-calibrated three PET/CT systems ((1) Biograph mCT S20: Siemens Medical Solutions, Erlangen, Germany; (2) Biograph 16: Siemens Medical Solutions; and (3) Discovery PET/CT 600: GE Healthcare, Pewaukee, WI, USA). These systems equipped a PET scanner and a multi-detector-row CT scanner (16 detectors). Low-dose CT with shallow breathing was firstly performed initially and used for both attenuation correction and also image fusion. Low-dose CT data for the Biograph mCT S20 was acquired at $120 \mathrm{kVp}$ using an auto-exposure-control system, and a beam pitch of 0.8 , slice thickness of $5 \mathrm{~mm}$. That data for the Biograph 16 was acquired at $120 \mathrm{kVp}$ using an auto-exposure-control system, beam pitch of 0.833 , and a slice thickness of $5 \mathrm{~mm}$. Finally, that data for the Discovery PET/CT 600 was acquired at $120 \mathrm{kVp}$ using an auto-exposure-control system, beam pitch of 0.938 , and a slice thickness of $3.75 \mathrm{~mm}$. Emission images were acquired in three-dimensional mode (Biograph mCT S20: $3.0 \mathrm{~min}$ per bed position; Biograph 16: $2.5 \mathrm{~min}$ per bed position; and Discovery PET/CT 600: 2.5 min per bed position). PET data were reconstructed with an ordered subset expectation maximization (OSEM) algorithm. It employed 3 iterations and 16 subsets for the Discovery PET/CT 600; 3 iterations, 8 subsets for the Biograph 16, and 2 iterations; and 21 subsets combined with time of fright for the Biograph mCT S20. A Gaussian filter was used for post-smoothing filter in all cases. It had a full-width at half-maximum of $5 \mathrm{~mm}$. For each patient, the same scanner was used for pre- and post-therapeutic PET/CT.

\section{FDG-PET/CT examination}

An in-house cyclotron and automated synthesis system (F200; Sumitomo Heavy Industries, Shinagawa, Tokyo, Japan) was used on the basis of the authorized procedure to synthesize FDG. Patients were instructed to fast for at least $5 \mathrm{~h}$ before intravenous injection of FDG, fixed at 5.0 $\mathrm{MBq} / \mathrm{kg}$. PET/CT images were acquired $60 \mathrm{~min}$ after injection. They were acquired with the same PET/CT scanner and manner as were the 4DST PET/CT images.

\section{Image analysis}

All 4DST and FDG PET/CT images were analyzed by two board-certified nuclear medicine physicians who were unaware of the clinical data. The primary tumor was defined as the volume of interest (VOI) and delineated on the 4DST and FDG PET scans using 
semi-automatic gradient-based delineation software (MIM Software, Cleveland [OH], USA). Its superiority over manual and threshold methods has been validated [18]. The following PET quantitative values were evaluated for the primary tumor: maximum standardized uptake value (SUVmax), metabolic tumor volume (MTV), total lesion glycolysis (TLG), and total lesion proliferation (TLP). The MTV was calculated by summing up the areas within each two-dimensional transverse tumor contour multiplied by the corresponding slice thickness automatically by the software. The TLG was calculated by multiplying MTV by SUVmean in the FDG study and the TLP in the same way for the 4DST study. Each parameter was assessed both pre-therapy (preSUVmax, ${ }_{\text {pre }} \mathrm{MTV}$, pre TLG, and ${ }_{\text {pre }} \mathrm{TLP}$ ) and post-therapy ( ${ }_{\text {post }} \mathrm{SUV}$ max, post MTV, post TLG, and post TLP), and then percent changes calculated [for SUVmax ( $\triangle$ SUVmax) for MTV $(\triangle \mathrm{MTV})$, for TLG $(\triangle \mathrm{TLG})$, and for TLP $(\Delta \mathrm{TLP})$. With FDG PET, tumor response was also evaluated using PET Response Criteria in Solid Tumors (PERCIST) version 1.0. [19]. The lean body mass-corrected SUV peak was measured by MIM Software.

\section{Histopathologic assessment}

The reference standard for the diagnosis of response to neoadjuvant therapy was histopathologic examination of the resected primary tumor specimen, which was performed by an experienced pathologist based on hematoxylin and eosin-stained tissue sections. The degree of pathologic response to neoadjuvant treatment was graded as follows [20]: (grade 0, ineffective) no recognizable histological therapeutic effect, (grade 1, slightly effective) apparently viable cancer cells account for $1 / 3$ or more of the tumor tissue, but there is some evidence of degeneration of the cancer tissue or cells grade 1a: viable cancer cells account for $2 / 3$ or more tumor tissue. Grade 1b: viable cancer cells account for $1 / 3$ or more, but $<2 / 3$, of tumor tissue, (grade 2 ; moderately effective) viable cancer cells account for less than $1 / 3$ of tumor tissue, (grade 3; markedly effective) no viable cancer cells. Patients who showed a grade 0-1a pathologic response were considered non-responders, while patients who were classified as grade $1 \mathrm{~b}-3$ were considered responders [21, 22].

\section{Statistical analysis}

No power analysis was performed because of the lack of previous studies on the topic. Data are expressed as mean \pm SD. The association between clinical baseline characteristics and responders versus non-responders was studied using Student's $T$ test for parametric continuous parameters, Mann-Whitney $U$ test for non-parametric continuous parameters, and Fisher's exact test for categorical parameters. Mann-Whitney $U$ test was used to compare quantitative values of 4DST and FDG PET parameters between the groups (responder and non-responder). Receiver operating curve (ROC) analyses (providing area-under-the-curve (AUC) values) were performed to evaluate the diagnostic ability of the FDG and 4DST PET parameters to distinguish responders from non-responders. Statistical significance was considered to be present for values of $\mathrm{p}$ less than 0.05 .

\section{Results}

\section{Clinical data}

The pathological diagnosis revealed 10 non-responders (grade 0: 1, grade 1a: 9) and 16 responders (grade 1b: 4, grade 2: 9, and grade 3: 3 ). The clinical characteristics of responders and non-responders are described in Table 2. None of the baseline characteristics, including tumor markers, were related to the pathologic response to neoadjuvant therapy. The mean interval between pretreatment FDG PET and pre-treatment 4DST PET and between post-treatment FDG PET and post-treatment 4DST PET was $4.7 \pm 5.2$ and $5.7 \pm 4.4$ days, respectively.

Table 2 Clinicopathologic characteristics comparing between responders and non-responders

\begin{tabular}{|c|c|c|c|}
\hline & Responders & Non-responders & $\overline{p \text { value }}$ \\
\hline No. patients & 16 & 10 & \\
\hline Sex, male & 14 & 9 & 1.00 \\
\hline $\begin{array}{l}\text { Mean age years (standard } \\
\text { deviation) }\end{array}$ & $65.2(10.7)$ & $71.6(4.9)$ & 0.072 \\
\hline Location & & & 0.17 \\
\hline Upper & 3 & 4 & \\
\hline Middle & 8 & 6 & \\
\hline Lower & 5 & 0 & \\
\hline Clinical T-stage & & & 0.141 \\
\hline cT2 & 1 & 1 & \\
\hline cT3 & 6 & 7 & \\
\hline cT4 & 9 & 2 & \\
\hline Clinical N-stage & & & 0.090 \\
\hline cNO & 1 & 5 & \\
\hline $\mathrm{cN} 1$ & 4 & 2 & \\
\hline $\mathrm{cN} 2$ & 10 & 3 & \\
\hline cN3 & 1 & 0 & \\
\hline Neoadjuvant therapy & & & 0.051 \\
\hline Chemotherapy & 6 & 8 & \\
\hline Chemoradiation therapy & 10 & 2 & \\
\hline \multicolumn{4}{|l|}{ Tumor marker } \\
\hline $\begin{array}{l}\mathrm{SCC}(\mathrm{ng} / \mathrm{mL}) \\
\text { [median (range)] }\end{array}$ & $1.9(0.7-6.2)$ & $2.8(0.6-8.1)$ & 0.36 \\
\hline $\begin{array}{l}\text { CEA (ng/mL) } \\
\text { [median (range)] }\end{array}$ & $2.4(0.6-6.4)$ & $2.1(0.2-8.6)$ & 0.90 \\
\hline
\end{tabular}


The mean interval between esophagectomy and post-treatment FDG PET and between esophagectomy and post-treatment 4DST PET was $14.4 \pm 6.7$ and $9.0 \pm$ 7.0 days, respectively. Thirteen patients underwent $\mathrm{PET} /$ CT with Discovery PET/CT 600, 6 patients with Biograph 16 and 7 patients with Biograph mCT S20.

\section{PET/CT parameters}

Table 3 demonstrates the values of parameters of preand post-therapeutic FDG and 4DST PET, and their percent changes, comparing between non-responders and responders. In post-therapeutic PET, 4DST post SUVmax was statistically lower for responders than those for non-responders $(p=0.001)$. As for percent changes of PET parameters between pre- and post-neoadjuvant therapy, FDG $\triangle$ SUVmax, $\Delta$ TLG, 4DST $\triangle$ SUVmax, and $\triangle T L P$ showed statistically greater reduction in responders than in non-responders. The AUCs of these PET parameters for discrimination of responders from non-responders are shown in Table 4, and ROC curve comparing between FDG $\triangle$ SUVmax and 4DST $\triangle \mathrm{SUV}$ max are described in Fig. 2. The representative case is demonstrated in Fig. 3.

Table 3 FDG and 4DST PET parameters pre- and post-neoadjuvant therapy, comparing between responders and non-responders (median, (interquartile range))

\begin{tabular}{|c|c|c|c|}
\hline Parameters & Responders & Non-responders & $p$ value \\
\hline \multicolumn{4}{|l|}{$\overline{\mathrm{FDG}}$} \\
\hline preSUVmax & $16.9(13.1,19.5)$ & $10.0(8.0,13.9)$ & 0.018 \\
\hline post $S U V \max$ & $4.5(3.2,5.4)$ & $6.1(4.2,7.9)$ & 0.11 \\
\hline$\Delta S U V \max (\%)$ & $-72.6(-78.4,67.2)$ & $-36.3(-49.9,-18.7)$ & $<0.001$ \\
\hline preMTV & $18.7(7.09,32.9)$ & $8.7(4.6,25.3)$ & 0.29 \\
\hline post MTV & $3.1(2.1,6.9)$ & $2.5(1.8,5.6)$ & 0.75 \\
\hline$\Delta \mathrm{MTV}(\%)$ & $-70.4(-86.1,-60.6)$ & $-59.1(-76.2,-45.7)$ & 0.27 \\
\hline pre ${ }^{T L G}$ & $150.5(70.8,249.8)$ & $38.3(19.6,160.8)$ & 0.14 \\
\hline post ${ }^{T L G}$ & $8.7(5.4,23.7)$ & $12.8(6.3,23.5)$ & 0.60 \\
\hline$\Delta \mathrm{TLG}(\%)$ & $-90.2(-95.1,-86.2)$ & $-65.3(-82.7,-58.2)$ & 0.020 \\
\hline \multicolumn{4}{|l|}{ 4DST } \\
\hline preSUVmax & $9.2(5.9,10.3)$ & $6.8(4.8,8.5)$ & 0.21 \\
\hline post SUVmax & $3.3(2.9,5.2)$ & $6.7(5.7,7.8)$ & 0.001 \\
\hline$\Delta$ SUVmax (\%) & $-56.7(-65.6,-40.8)$ & $-2.9(-10.5,15.9)$ & $<0.001$ \\
\hline preMTV & $17.8(9.7,36.9)$ & $15.1(3.4,34.9)$ & 0.53 \\
\hline postMTV & $2.1(1.4,3.6)$ & $4.0(1.6,10.5)$ & 0.43 \\
\hline$\Delta \mathrm{MTV}(\%)$ & $-86.9(-92.0,-72.9)$ & $-54.3(-75.1,-25.1)$ & 0.11 \\
\hline preTLP & $66.9(37.1,201.9)$ & $48.8(15.5,88.0)$ & 0.34 \\
\hline post $T L P$ & $5.3(2.9,10.9)$ & $11.9(6.5,34.1)$ & 0.102 \\
\hline$\Delta \mathrm{TLP}(\%)$ & $-91.6(-95.1,-87.0)$ & $-54.4(-75.6,-2.3)$ & 0.020 \\
\hline
\end{tabular}

SUVmax maximum standardized uptake value, MTV metabolic tumor volume, $T L G$ total lesion glycolysis, TLP total lesion proliferation
PERCIST 1.0 criteria provided diagnostic ability for discriminating responders with sensitivity of 1.000 , specificity of 0.727 , accuracy of 0.769 , positive predictive value (PPV) of 0.400 , and negative predictive value (NPV) of 1.000, when patients with partial metabolic response (PMR) and complete metabolic response (CMR) are defined as patients with response, and patients with stable metabolic disease (SMD) and progressive metabolic disease (PMD) are considered as patients without response.

\section{Discussion}

The purpose of this study was to investigate whether 4DST-PET is useful for predicting the treatment response in patients with esophageal cancer, compared to the usefulness of FDG-PET. 4DST $\triangle$ SUVmax provided high AUC values to distinguish treatment responders among all PET parameters. In addition, 4DST post SUVmax was also helpful for predicting response.

While FDG post SUVmax was not statistically different between responders and non-responders, $4 \mathrm{DST}{ }_{\text {post }} \mathrm{SUV}$ max were statistically higher for responders and showed great diagnostic performance in the discrimination of responders $(A U C=0.88)$. It has been reported that post-therapeutic FDG-PET is useful for evaluation of tumor response in esophageal cancer [23-25], which is concordant with our results. A possible explanation why 4DST post SUVmax showed greater diagnostic value than that of FDG is that FDG accumulation can be affected by inflammation due to chemotherapy or chemoradiation therapy [26, 27], whereas the influence of these therapies for 4DST may not be as significant. Indeed, high tumor selectivity of 4DST, which enables discrimination between tumor and inflammation, has been demonstrated in the rodent model [28]. The other possible reason is that 4DST simply measures tumor proliferation more accurately than FDG does. 4DST uptake corresponded well to Ki-67 [29], and in lung cancer, Minamimoto et al. have reported 4DST shows a better correlation with Ki-67 than FDG does [13]. Therefore, 4DST may have the potential to reflect tumor viability more precisely than FDG, not only in lung cancer but also in esophageal cancer. The higher accumulation of 4DST is likely to indicate more residual viable cancer cells.

Both FDG $\triangle$ SUVmax and 4DST $\triangle$ SUVmax were useful for evaluating response, and PERCIST 1.0 also provided good diagnostic performance, whereas 4DST $\triangle$ SUVmax showed higher accuracy than PRECIST 1.0. It has been reported that percent changes of FDG uptake (including PERCIST 1.0) between pre- and post-treatment are effective for the evaluation of response in esophageal cancer [21, 23, 30]. In PERCIST 1.0, the cutoff value for the response is set at more than or equal to $30 \%$. However, several studies suggested optimal cutoff 
Table 4 ROC analysis for discriminating responders from non-responders using FDG and 4DST PET parameters

\begin{tabular}{|c|c|c|c|c|c|c|c|}
\hline Parameters & AUC $(95 \% \mathrm{Cl})$ & Optimal cutoff value & Sensitivity & Specificity & Accuracy & PPV & NPV \\
\hline \multicolumn{8}{|l|}{ FDG } \\
\hline preSUVmax & $0.78(0.58-0.98)$ & $\geq 10.7$ & 0.600 & 0.938 & 0.808 & 0.857 & 0.789 \\
\hline$\Delta S U V \max (\%)$ & $0.92(0.78-1.00)$ & $\leq-60.3$ & 0.900 & 0.875 & 0.885 & 0.818 & 0.933 \\
\hline$\Delta \mathrm{TLG}(\%)$ & $0.78(0.56-0.99)$ & $\leq-84.7$ & 0.800 & 0.812 & 0.808 & 0.727 & 0.867 \\
\hline \multicolumn{8}{|l|}{ 4DST } \\
\hline post $S U V \max$ & $0.88(0.75-1.00)$ & $\leq 4.00$ & 1.000 & 0.625 & 0.769 & 0.625 & 1.000 \\
\hline$\Delta S U V \max (\%)$ & $0.92(0.80-1.00)$ & $\leq-19.5$ & 0.900 & 0.938 & 0.923 & 0.900 & 0.938 \\
\hline$\Delta \mathrm{TLP}(\%)$ & $0.78(0.57-0.99)$ & $\leq-86.7$ & 0.800 & 0.812 & 0.808 & 0.727 & 0.867 \\
\hline
\end{tabular}

SUVmax maximum standardized uptake value, MTV metabolic tumor volume, TLG total lesion glycolysis, TLP total lesion proliferation, $P P V$ positive predictive value, NPV negative predictive value

values for response evaluation in esophageal cancer as high as $50-60 \%[21,23,31]$. This percentage was similar to optimal cutoff values of FDG $\triangle$ SUVmax in our study (60.3\%), which showed higher diagnostic performance than when using $30 \%$ as a cutoff value. Indeed, it is frequently difficult to determine the optimal cutoff value in esophageal cancer, because non-responders also tend to show reduction of FDG accumulation [22, 23, 30]. In fact, the reduction rate of FDG $\triangle S U V \max$ in non-responder was not small (median FDG $\triangle$ SUVmax $=-36.3 \%)$ in this study as well. Conversely, in 4DST PET, non-responders showed almost no change from baseline PET, whereas responders showed a great reduction of uptake (median 4DST $\triangle$ SUVmax: nonresponder $=-2.9 \%$, responder $=-56.7 \%$ ). These results indicate that persistent 4DST uptake into primary lesion after treatment is highly suggestive of inadequate response. As such, the percent changes of 4DST accumulation are likely to be an easy-to-use marker in clinical practice.

FDG preSUVmax was higher for responders than for non-responders, which was statistically significant $(p=0.018)$. Although various studies have failed to demonstrate the prognostic value of the baseline SUVmax in FDG-PET [26, 32-34], some reported higher FDG SUVmax in initial PET correlated with a

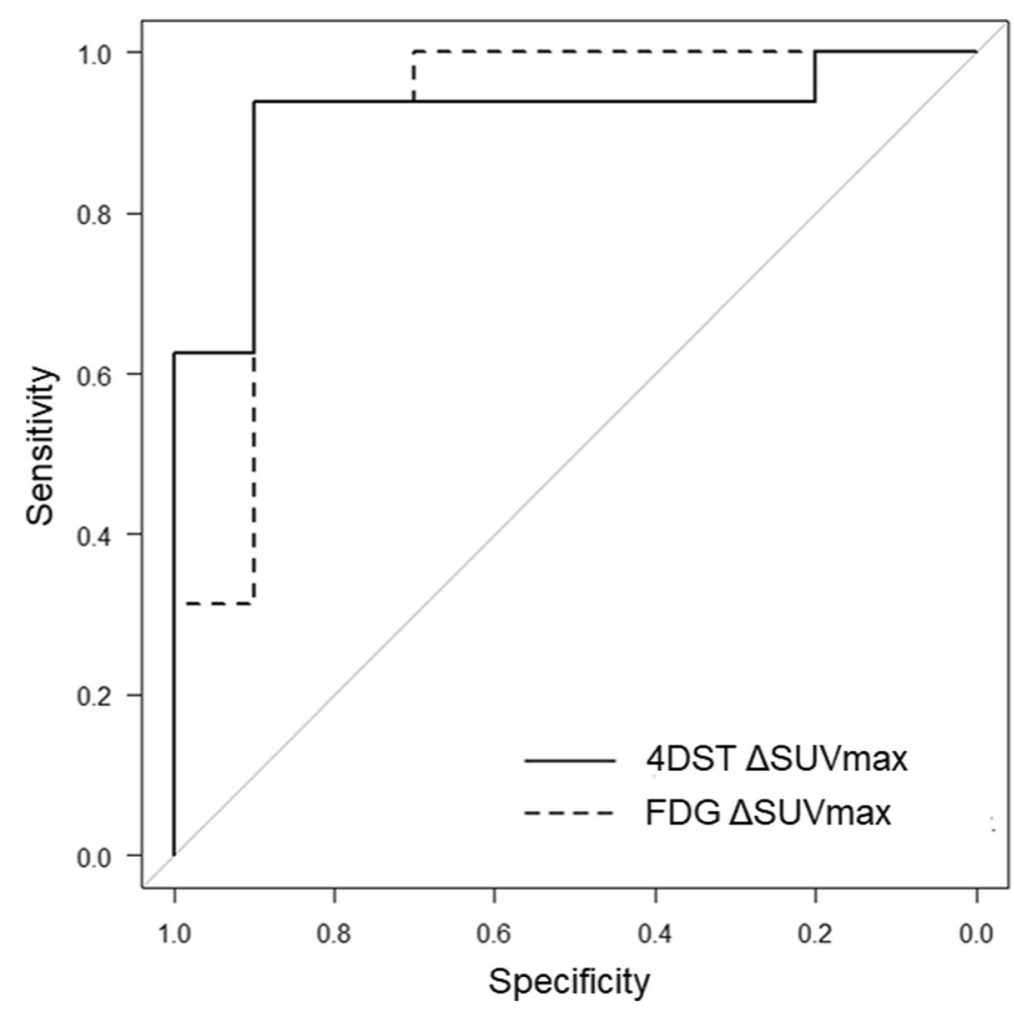

Fig. 2 Receiver operating characteristic curve comparing between 4DST $\triangle$ SUVmax and FDG $\Delta$ SUVmax 


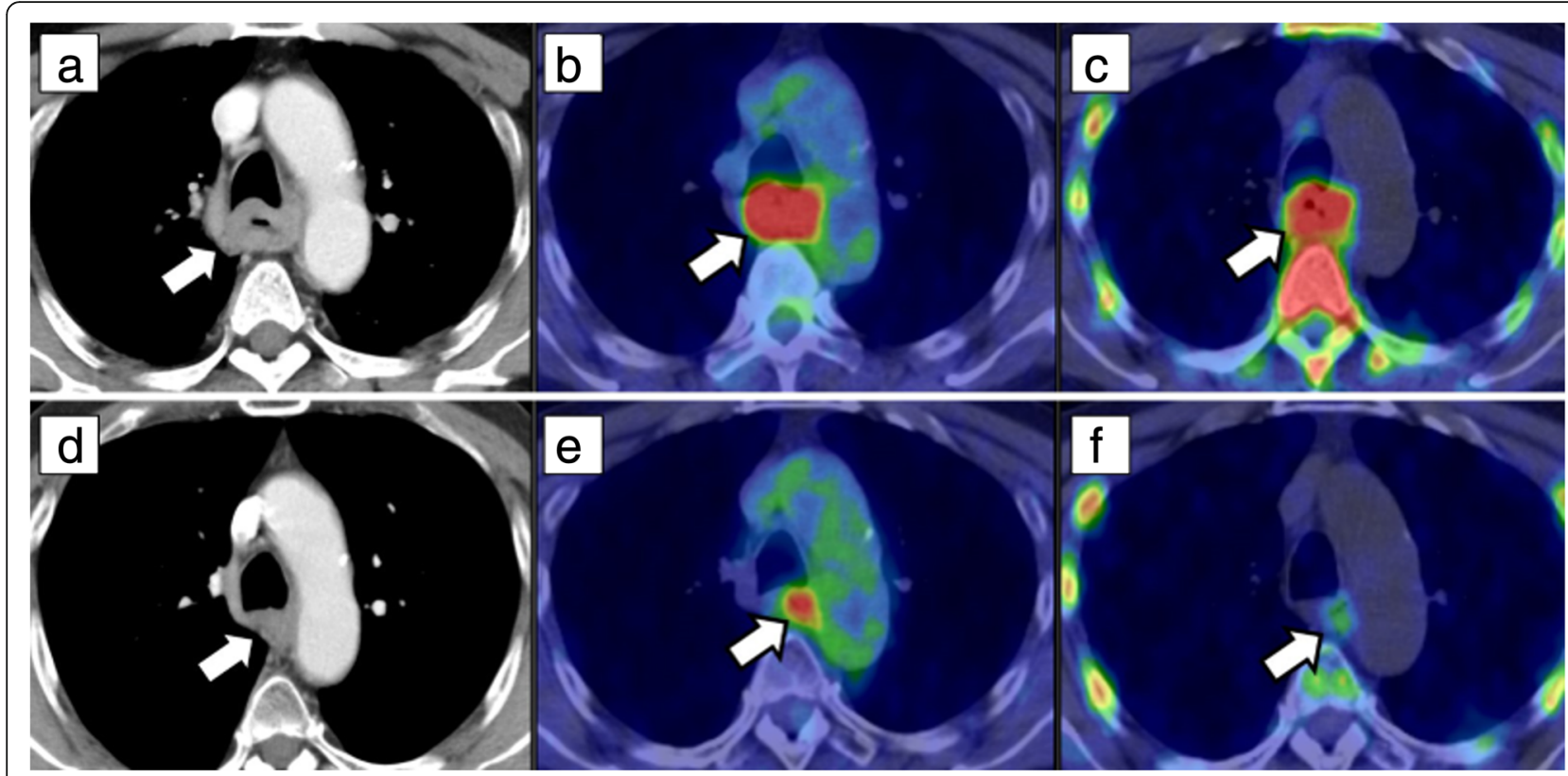

Fig. 3 A 68-year-old man with esophageal cancer treated by neoadjuvant chemoradiation therapy followed by esophagectomy. Baseline images (CT (a), FDG-PET/CT (b), and 4DST-PET/CT (c)). Post-neoadjuvant therapy images (CT (d), FDG-PET/CT (e), and 4DST-PET/CT (f)). Baseline chest CT showed esophageal cancer (a: arrow) in the upper thoracic region, and FDG and 4DST PET/CT demonstrated uptake into the primary lesion (b, c: arrow). Maximum standardized uptake value (SUVmax) of FDG and 4DST was 13.5 and 6.2, respectively. After neoadjuvant chemoradiation therapy, the tumor showed reduction in size (d: arrow). Post-therapeutic FDG-PET/CT demonstrated FDG-avid (SUVmax $=5.3$ ) primary lesion (e: arrow) suggesting the possibility of inadequate response. In contrast, 4DST-PET/CT showed relatively low uptake (SUVmax $=2.5$ ) into the tumor (f: arrow) indicating this patient was a responder. Subsequently, the patient underwent esophagectomy and pathologically confirmed as responder status (grade 2)

higher rate of complete histological response [35], and even with better disease-free survival [36]. Some suggested this is because higher FDG SUVmax is related to higher proliferation cells that are rapidly proliferating may better respond to chemotherapy or chemoradiation therapy [37]. However, if this theory is true, 4DST preSUVmax of responders should have been statistically higher than that of non-responders. This point should be discussed in future studies.

Further, based on $\triangle$ SUVmax, the ability for the differentiation of responders from non-responders were almost identical as depicted in ROC (Fig. 2); however, a few cases showed discrepancy between FDG and 4DST $\Delta \operatorname{SUVmax}(n=2)$. As discussed earlier, a possible explanation for this discrepancy could be that FDG accumulation can be affected by inflammation due to chemotherapy or radiation therapy, whereas the influence of these therapies for 4DST may not be significant. This issue needs to be discussed in further studies with large sample size.

This is the first study to report the utility of 4DST for the evaluation of therapeutic response in malignancy. Gerbaudo et al. has reported the efficacy of FLT, of which biodistribution is like 4DST [17], for the evaluation of treatment response in esophageal cancer [38]. Although the number of patients was limited in their study, the authors first showed that in patients with esophageal adenocarcinomas, FLT-PET demonstrated early therapy-induced decrease in tumor proliferation in response to treatment. The difference in the reduction in tumor FLT uptake during treatment between responders and non-responders was statistically significant, but for FDG, it was not. This was probably due to the fact that FLT uptake was not affected as much as FDG was by radiation induced inflammation. On the other hand, the difference in the reduction in tumor FLT uptake at after completion of therapy between responders and non-responders was no longer significant. Gerbaudo and colleagues explained that at the end of chemoradiation treatment, FLT uptake could have been affected by the continuous effect of radiation, which diminished the proliferative capacity of remaining viable cells. In contrast, post $S U V \max$ and $\triangle \mathrm{SUVmax}$ in $4 \mathrm{DST}$ were useful to distinguish responders from nonresponders at the end of therapy in our study. The advantage of 4DST over FLT in proliferation measurement is presented in vivo analysis [10]. Namely, FLT is not incorporated into DNA and reflects salvage pathway of DNA synthesis [12], whereas 4DST incorporates into DNA directly [9]. This feature may be one of the possible reasons to explain the discrepancy of usefulness between 4DST and FLT-PET in the assessment of 
treatment response. The other reason might be that more than half of the patients in our study were not treated with radiation. Considering Gerbaudo et al. findings described above about the use of interim FLT-PET for early therapeutic monitoring in esophageal cancer, it is probable that interim 4DST-PET will also have great diagnostic potential in this setting and should be examined in future studies.

There are some limitations in this study. Firstly, it was performed in a single center with a relatively small number of patients. Larger prospective multicenter trials are necessary in the future. Secondly, the mixed population of patients in terms of neoadjuvant therapy (chemotherapy vs. chemoradiation therapy) is another limitation, but this factor was not statistically significant between responders and non-responders. Thirdly, three PET scanners were used in this study, which potentially influenced the values of the PET parameters. However, cross-calibration between the three scanners was performed, and the same scanner was used in each patient not only for pre- and post-neoadjuvant therapy scan but also for 4DST and FDG PET scan. Thus, the influence of the difference of scanners is considered minimal. Finally, a cyclotron is necessary for the production of 4DST, which may be a drawback for this C11-labeled PET tracer.

\section{Conclusions}

$\triangle S U V \max$ and ${ }_{\text {post }}$ SUVmax in $4 \mathrm{DST}$ PET can provide great diagnostic value for discriminating responders from non-responders in patients with esophageal cancer. A persistent 4DST uptake after neoadjuvant therapy strongly suggests the presence of residual viable tumor cells. 4DST PET showed a great potential for evaluation of treatment response in esophageal cancer, of which assessment is higher than that of FDG PET. This study was the first report to represent the usefulness of 4DST in evaluating therapeutic response and will perhaps stimulate future research that investigates the utility of 4DST in malignancies other than esophageal cancer.

\footnotetext{
Abbreviations

4DST: 4'-[Methyl-11C] thiothymidine; AUC: Area-under-the-curve; CMR: Complete metabolic response; FLT: 3'-Fluoro-3'-deoxythymidine; MTV: Metabolic tumor volume; NPV: Negative predictive value; OSEM: Ordered-subset expectation maximization; PERCIST: PET Response Criteria in Solid Tumors; PMD: Progressive metabolic disease; PMR: Partial metabolic response; PPV: Positive predictive value; ROC: Receiver operating curve; SUV: Standardized uptake value; TLG: Total lesion glycolysis; TLP: Total lesion proliferation; VOI: Volume of interest
}

\section{Acknowledgements}

The authors thank the Department of Pathology, National Center for Global Health and Medicine, for pathological examinations of surgical specimens.

\section{Funding}

This work was supported by a Grant from a Grant-in Aid for Young Scientists (B) (No. 17K18396) from the Japan Society for the Promotion of Science (to Masatoshi Hotta) and a Bayer Best Research Award 2017 (No. KJ-06) from the
Japan Radiological Society (to Masatoshi Hotta). No other potential conflict of interest relevant to this article was reported.

\section{Availability of data and materials}

The datasets used and/or analysed during the current study are available from the corresponding author on reasonable request.

\section{Authors' contributions}

All authors provided substantial contributions to the conception or design of the work. MH, RM, KY, KN, and KT contributed to the acquisition, analysis, or interpretation of data for the work. RM, KY, KN, DS, KN, DS, and KT helped in drafting the work or revising it critically for important intellectual content. All authors approved the final version to be published and agreed to be accountable for all aspects of the work in ensuring that questions related to the accuracy or integrity of any part of the work are appropriately investigated and resolved.

\section{Authors' information}

Part of this study was presented at the 2018 SNMMl Meeting ( $J$ Nucl Med May 1, 2018 vol. 59 no. supplement 1158).

Ethics approval and consent to participate

This study was approved by the ethical committee at National Center for Global Health and Medicine.

All procedures performed in studies involving human participants were in accordance with the ethical standards of the institutional and/or national research committee and with the 1964 Helsinki declaration and its later amendments or comparable ethical standards.

\section{Consent for publication}

Written informed consents were obtained from all subjects for participation and publication of this report.

\section{Competing interests}

The authors declare that they have no competing interests.

\section{Publisher's Note}

Springer Nature remains neutral with regard to jurisdictional claims in published maps and institutional affiliations.

\section{Author details}

${ }^{1}$ Division of Nuclear Medicine, Department of Radiology, National Center for Global Health and Medicine, 1-21-1, Toyama, Shinjuku-ku, Tokyo 162-8655, Japan. ${ }^{2}$ Department of Surgery, National Center for Global Health and Medicine, 1-21-1, Toyama, Shinjuku-ku, Tokyo 162-8655, Japan. ${ }^{3}$ Functional Brain Research Team for Neuroimaging, Tokyo Metropolitan Institute of Gerontology, 35-2, Sakae-cho, Itabashi-ku, Tokyo 173-0015, Japan. ${ }^{4}$ Department of Diagnostic Radiology, Tohoku University Hospital, 1-1, Seiryo-machi, Aoba-ku, Sendai, Miyagi 980-8574, Japan.

Received: 13 November 2018 Accepted: 18 January 2019 Published online: 30 January 2019

\section{References}

1. Gebski V, Burmeister B, Smithers BM, et al. Survival benefits from neoadjuvant chemoradiotherapy or chemotherapy in oesophageal carcinoma: a meta-analysis. Lancet Oncol. 2007;8:226-34.

2. Sjoquist KM, Burmeister BH, Smithers BM, et al. Survival after neoadjuvant chemotherapy or chemoradiotherapy for resectable oesophageal carcinoma: an updated meta-analysis. Lancet Oncol. 2011;12:681-92.

3. NCCN Clinical practice guidelines in oncology (NCCN guidelines)_esophageal and esophagogastric junction cancers version 2 , 2018. Available from: http://www.nccn.org/professionals/physician_gls/pdf/ esophageal.pdf. Accessed 18 June 2018.

4. Brucher BL, Becker K, Lordick F, et al. The clinical impact of histopathologic response assessment by residual tumor cell quantification in esophageal squamous cell carcinomas. Cancer. 2006;106:2119-27.

5. Tong DK, Law S, Kwong DL, et al. Histological regression of squamous esophageal carcinoma assessed by percentage of residual viable cells after neoadjuvant chemoradiation is an important prognostic factor. Ann Surg Oncol. 2010;17:2184-92. 
6. Kwee RM. Prediction of tumor response to neoadjuvant therapy in patients with esophageal cancer with use of 18 F FDG PET: a systematic review. Radiology. 2010;254:707-17.

7. Klaeser B, Nitzsche E, Schuller JC, et al. Limited predictive value of FDG-PET for response assessment in the preoperative treatment of esophageal cancer: results of a prospective multi-center trial (SAKK 75/02). Onkologie. 2009;32:724-30.

8. van Heijl M, Omloo JM, van Berge Henegouwen Ml, et al. Fluorodeoxyglucose positron emission tomography for evaluating early response during neoadjuvant chemoradiotherapy in patients with potentially curable esophageal cancer. Ann Surg. 2011;253:56-63.

9. Toyohara J, Kumata K, Fukushi K, et al. Evaluation of 4'-[methyl-14C] thiothymidine for in vivo DNA synthesis imaging. J Nucl Med. 2006;47:1717-22.

10. Toyohara J, Okada M, Toramatsu C, et al. Feasibility studies of 4'-[methyl(11)C] thiothymidine as a tumor proliferation imaging agent in mice. Nucl Med Biol. 2008;35:67-74.

11. Plotnik DA, Wu S, Linn GR, et al. In vitro analysis of transport and metabolism of 4'-thiothymidine in human tumor cells. Nucl Med Biol. 2015;42:470-4.

12. Been LB, Suurmeijer AJ, Cobben DC, et al. [18F]FLT-PET in oncology: current status and opportunities. Eur J Nucl Med Mol Imaging. 2004:31:1659-72.

13. Minamimoto R, Toyohara J, Seike A, et al. 4'-[Methyl-11C]-thiothymidine PET/CT for proliferation imaging in non-small cell lung cancer. J Nucl Med. 2012;53:199-206.

14. Minamimoto R, Toyohara J, Ito H, et al. A pilot study of 4'-[methyl-11C]thiothymidine PET/CT for detection of regional lymph node metastasis in non-small cell lung cancer. EJNMMI Res. 2014;4:10.

15. Minamimoto R, Nakaigawa N, Nagashima Y, et al. Comparison of 11C-4DST and 18F-FDG PET/CT imaging for advanced renal cell carcinoma: preliminary study. Abdom Radiol (NY). 2016;41:521-30.

16. Hoshikawa H, Mori T, Maeda Y, et al. Influence of volumetric 4'-[methyl(11)C]-thiothymidine PET/CT parameters for prediction of the clinical outcome of head and neck cancer patients. Ann Nucl Med. 2017;31:63-70.

17. Toyohara J, Nariai T, Sakata M, et al. Whole-body distribution and brain tumor imaging with (11)C-4DST: a pilot study. J Nucl Med. 2011;52:1322-8.

18. Werner-Wasik M, Nelson AD, Choi W, et al. What is the best way to contour lung tumors on PET scans? Multiobserver validation of a gradient-based method using a NSCLC digital PET phantom. Int J Radiat Oncol Biol Phys. 2012;82:1164-71.

19. Wahl RL, Jacene H, Kasamon $Y$, et al. From RECIST to PERCIST: evolving considerations for PET response criteria in solid tumors. J Nucl Med. 2009; 50(Suppl 1):122s-50s.

20. Japan Esophageal Society. Japanese classification of esophageal cancer, 11th edition: part I. Esophagus. 2017;14:1-36.

21. Odawara S, Kitajima K, Katsuura T, et al. Tumor response to neoadjuvant chemotherapy in patients with esophageal cancer assessed with CT and FDG-PET/CT - RECIST 1.1 vs. PERCIST 1.0. Eur J Radiol. 2018;101:65-71.

22. Tani $Y$, Nakajima M, Kikuchi M, et al. (1)(8)F-Fluorodeoxyglucose positron emission tomography for evaluating the response to neoadjuvant chemotherapy in advanced esophageal cancer. Anticancer Res. 2016;36:367-73.

23. Sasaki K, Uchikado Y, Okumura H, et al. Role of (18)F-FDG-PET/CT in esophageal squamous cell carcinoma after neoadjuvant chemoradiotherapy. Anticancer Res. 2017;37:859-64.

24. Hamai Y, Hihara J, Emi M, et al. Ability of fluorine-18 fluorodeoxyglucose positron emission tomography to predict outcomes of neoadjuvant chemoradiotherapy followed by surgical treatment for esophageal squamous cell carcinoma. Ann Thorac Surg. 2016:102:1132-9.

25. Cong L, Wang S, Gao T, et al. The predictive value of 18F-FDG PET for pathological response of primary tumor in patients with esophageal cancer during or after neoadjuvant chemoradiotherapy: a meta-analysis. Jpn J Clin Oncol. 2016;46:1118-26.

26. Wieder HA, Brucher BL, Zimmermann F, et al. Time course of tumor metabolic activity during chemoradiotherapy of esophageal squamous cell carcinoma and response to treatment. J Clin Oncol. 2004:22:900-8.

27. Roedl JB, Colen RR, Holalkere NS, et al. Adenocarcinomas of the esophagus: response to chemoradiotherapy is associated with decrease of metabolic tumor volume as measured on PET-CT. Comparison to histopathologic and clinical response evaluation. Radiother Oncol. 2008;89:278-86.

28. Toyohara J, Elsinga PH, Ishiwata $\mathrm{K}$, et al. Evaluation of 4'-[methyl-11C] thiothymidine in a rodent tumor and inflammation model. J Nucl Med. 2012;53:488-94.
29. Toyohara J, Sakata M, Oda K, et al. Longitudinal observation of [11C]4DST uptake in turpentine-induced inflammatory tissue. Nucl Med Biol. 2013;40:240-4.

30. van Rossum PS, Fried DV, Zhang $L$, et al. The value of (18)F-FDG PET before and after induction chemotherapy for the early prediction of a poor pathologic response to subsequent preoperative chemoradiotherapy in oesophageal adenocarcinoma. Eur J Nucl Med Mol Imaging. 2017;44:71-80.

31. Makino T, Yamasaki M, Tanaka K, et al. Metabolic tumor volume change predicts long-term survival and histological response to preoperative chemotherapy in locally advanced esophageal cancer. Ann Surg. 2018. https://doi.org/10.1097/SLA.0000000000002808.

32. Omloo JM, van Heijl M, Hoekstra OS, et al. FDG-PET parameters as prognostic factor in esophageal cancer patients: a review. Ann Surg Oncol. 2011;18:3338-52.

33. Van de Wiele C, Kruse V, Smeets $P$, et al. Predictive and prognostic value of metabolic tumour volume and total lesion glycolysis in solid tumours. Eur J Nucl Med Mol Imaging. 2013;40:290-301.

34. Touboul E, Huguet F, Talbot JN. Use of PET for staging, treatment evaluation, and follow-up in esophageal cancers. Cancer Radiother. 2008; 12:633-9.

35. Levine EA, Farmer MR, Clark $P$, et al. Predictive value of 18-fluoro-deoxyglucose-positron emission tomography (18F-FDG-PET) in the identification of responders to chemoradiation therapy for the treatment of locally advanced esophageal cancer. Ann Surg. 2006:243:472-8.

36. Lemarignier C, Di Fiore F, Marre C, et al. Pretreatment metabolic tumour volume is predictive of disease-free survival and overall survival in patients with oesophageal squamous cell carcinoma. Eur J Nucl Med Mol Imaging. 2014;41:2008-16.

37. Vesselle H, Schmidt RA, Pugsley JM, et al. Lung cancer proliferation correlates with [F-18] fluorodeoxyglucose uptake by positron emission tomography. Clin Cancer Res. 2000;6:3837-44.

38. Gerbaudo VH, Killoran JH, Kim CK, et al. Pilot study of serial FLT and FDGPET/CT imaging to monitor response to neoadjuvant chemoradiotherapy of esophageal adenocarcinoma: correlation with histopathologic response. Ann Nucl Med. 2018;32:165-74.

\section{Submit your manuscript to a SpringerOpen ${ }^{\circ}$ journal and benefit from:}

- Convenient online submission

- Rigorous peer review

- Open access: articles freely available online

High visibility within the field

- Retaining the copyright to your article

Submit your next manuscript at $>$ springeropen.com 